Horizons philosophiques

\title{
Jean-Claude Guédon, Gutenberg Contemporain
}

\section{Éric Bernatchez}

Volume 6, numéro 2, printemps 1996

La philosophie sur Internet

URI : https://id.erudit.org/iderudit/801014ar

DOI : https://doi.org/10.7202/801014ar

Aller au sommaire du numéro

\section{Éditeur(s)}

Collège Édouard-Montpetit

\section{ISSN}

1181-9227 (imprimé)

1920-2954 (numérique)

Découvrir la revue

\section{Citer ce document}

Bernatchez, É. (1996). Jean-Claude Guédon, Gutenberg Contemporain. Horizons philosophiques, 6(2), 91-93. https://doi.org/10.7202/801014ar d'utilisation que vous pouvez consulter en ligne.

https://apropos.erudit.org/fr/usagers/politique-dutilisation/ 


\section{JEAN-CLAUDE GUÉDON ${ }^{1}$, GUTENBERG CONTEMPORAIN}

\section{Entrevue réalisée par Eric Bernatchez ${ }^{2}$}

Nous sommes peut-être en train d'entrer dans un turboMoyen-Âge, car l'enjeu essentiel de notre époque est de restituer à la communication la place qu'elle avait aux origines par l'oralités.

EB : Avec l'électronique, n'importe quel auteur peut être publié. Nous commençons déjà à le constater dans certains forums où l'on peut lire, entre autres, de la poésie d'un goût douteux. Quel effet l'édition électronique aura-t-elle sur la littérature?

Jean-Claude Guédon : L'imprimé s'est doté d'une respectabilité sociale en tant qu'objet. On a tendance à croire davantage ce qui est imprimé. Et il y a une sorte de promotion sociale dans le simple fait d'être imprimé, car c'est coûteux. Comme il s'agit d'une ressource rare, ceux qui arrivent à être publiés témoignent de ressources intellectuelles, institutionnelles ou financières. Dans le cas des journaux, il y a des gens qui ont un statut mondialement reconnu comme fournisseurs d'information. On les appelle «les journalistes». Ils sont un filtre social. Ils valident l'information, et on connaît leurs règles d'éthique, leurs droits et devoirs. La pratique journalistique est balisée. Dans le cas d'Internet, la situation change complètement. Regardez ce qui s'est passé récemment au Chiapas. Le souscommandant Marcos a simplement fait circuler sur Internet des récits d'atrocités que tout le monde a repris. Or, c'est de la rumeur. Le réseau Internet produit de la rumeur. II n'y a person-

1. Coresponsable du programme de la grande conférence INET 96, Jean-Luc Guédonn'est pas informaticien, encoremoins ingénieur. Il enseigne au département de littérature comparée de l'Université de Montréal. II a lancé la première revue savante électronique, Surfaces, à une époque où le W3 était inconnu.

2. pour Z-mag (http://planete.qc.ca/ Z)

3. Citation de Jean-Luc Guédon. 
ne pour vérifier ce qui y circule. C'est exactement comme ce qui m'est arrivé à Paris. J'étais dans le quartier Saint-Michel lors de l'attentat dans le RER. J'ai entendu toutes sortes de rumeurs pour expliquer ce qui s'était passé : suicide, incendie, etc. Rumeurs reprises par les journaux. Au fond, ce qu'on fait dans les journaux, ce n'est que domestiquer la rumeur, la valider. Sur Internet, on n'a pas encore trouvé de moyens efficaces de faire au moins la même chose. À mon avis, il faudra trouver des instances de validation de l'information sur Internet. Je ne sais pas quoi exactement, mais on peut imaginer des comités de lecteurs qui attribueraient des labels aux informations qui circulent, afin d'attester leur authenticité. Bien sûr, il ne pourrait s'agir d'une forme de contrôle. Les informations non-approuvées pourraient circuler quand même.

EB : On a souvent annoncé l'avènement de l'édition électronique pour demain. Est-il réaliste de croire que nous lirons un jour nos journaux en version électronique?

Jean-Claude Guédon : Je crois que l'électronique ne remplacera pas nécessairement le papier. II y a une règle classique en technologie qui veut qu'une nouvelle technologie ne remplace jamais l'ancienne. Elle la déplace et lui fait jouer de nouveaux rôles, plus limités ou marginaux. Lorsqu'on voit du texte sur un écran, notre première impression c'est que l'électronique a remplacé l'imprimé. Ce n'est pas vrai du tout. II n'a fait que nous forcer à repenser les rôles essentiels de l'imprimé. Pour certaines formes de lecture, l'imprimé est une technologie fabuleuse : portable et légère, la feuille de papier nous permet de lire tout en annotant les marges et en soulignant. Je crois que cela ne disparaîtra pas de sitôt.

En revanche, l'électronique servira surtout à faire des lectures rapides, en diagonale, des survols. Le lecteur n'imprimera que les extraits qu'il souhaite lire au complet. Nous avons d'ailleurs conçu Surfaces de façon à favoriser ce mode de lecture. Dans Surfaces, chaque article est un fichier distinct. Nous avons même des versions WordPerfect pour DOS et MSWord pour Mac, de façon à ce que le lecteur, en imprimant le 
texte, obtienne une mise en page comparable à celle qu'il aurait avec une revue scientifique imprimée. En quelque sorte, nous déléguons au lecteur l'impression de Surfaces. Et cela nous fait épargner environ $30 \%$ des frais habituels de production d'une revue scientifique!

EB : Le papier est portable, soit. Mais n'oublions pas que certains best-sellers pèsent des tonnes. Avecle développement des écrans à cristauxliquides, n'aurons-nous pas inévitablement, un jour, des «feuilles» électroniques?

Jean-Claude Guédon : C'est ce dont nous rêvons tous. William Gibson, l'auteur du mot «cyberspace», a déja répondu fort intelligemment à cette question. On lui demandait : "Que sera l'avenir du livre?» et il avait répliqué : "L'avenir du livre, c'est très simple. II s'agira d'un livre très très beau, un objet d'art magnifique. Et ce livre ne contiendra qu'une seule feuille sur laquelle nous ferons défiler toutes les pages l'une après l'autre".

Eric Bernatchez 